PROCEEDINGS OF THE

AMERICAN MATHEMATICAL SOCIETY

Volume 132, Number 5, Pages 1525-1529

S 0002-9939(03)07404-5

Article electronically published on December 23, 2003

\title{
THE CHEEGER CONSTANT OF SIMPLY CONNECTED, SOLVABLE LIE GROUPS
}

\author{
NORBERT PEYERIMHOFF AND EVANGELIA SAMIOU
}

(Communicated by Wolfgang Ziller)

\begin{abstract}
We show that the Cheeger isoperimetric constant of a solvable simply connected Lie group $G$ with Lie algebra $\mathfrak{G}$ is

$$
h(G)=\max _{H \in \mathfrak{G},\|H\|=1} \operatorname{tr}(\operatorname{ad}(H)) .
$$
\end{abstract}

\section{INTRODUCTION}

The Cheeger isoperimetric constant $h(M)$ of a complete noncompact Riemannian manifold $M$ is defined by

$$
h(M)=\inf _{K \subset M} \frac{\operatorname{area}(\partial K)}{\operatorname{vol}(K)},
$$

where $K$ ranges over all connected, open submanifolds of $M$ with compact closure and smooth boundary. For the bottom of the spectrum $\lambda(\Omega)$, there is Cheeger's inequality ([Che, $\mathrm{BPP}, \overline{\mathrm{Cha}})$

$$
\lambda(\Omega) \geq \frac{h(\Omega)^{2}}{4} .
$$

There is a converse of Cheeger's inequality due to Peter Buser, $\mathrm{Bu}, \mathrm{BPP}$ : There exist constants $c_{1}$ and $c_{2}$, depending on a lower bound on the Ricci curvature of $M$ such that

$$
\lambda \leq c_{1} h+c_{2} h^{2} .
$$

Further relations between isometric and spectral properties (in particular, estimates of the heat kernel) can be found in the work of, e.g., Varopoulos, Coulhon, Saloff-Coste, Grigor'yan [Gr] and Pittet [Pi]. For connections between the Cheeger constant and Kazhdan's property T, see, e.g., $[\mathrm{Br}]$ or $[\mathrm{Leu}]$.

If $h(M)>0$, one can easily see that $M$ has exponential volume growth. The converse is not true. Hoke [Ho] has shown that $h(G)=0$ for a simply connected Lie group with left-invariant metric if and only if $G$ is unimodular and amenable. These Lie groups, however, have exponential volume growth if they are not of type R, see Pa]. Examples of this class are horospheres in symmetric spaces of noncompact type and higher rank orthogonal to the barycenter of a Weyl chamber, see [Pe].

In this note we calculate the Cheeger constant for simply connected solvable Lie groups.

Received by the editors November 6, 2001.

2000 Mathematics Subject Classification. Primary 53C30, 22E25.

(C)2003 American Mathematical Society 


\section{The Cheeger constant}

We consider the following situation: Let $G=G_{0} \rtimes \mathbb{R}$ be a semidirect product with Lie algebra $\mathfrak{G}=\mathfrak{G}_{0} \oplus \mathbb{R}$ endowed with a left-invariant metric such that $\mathfrak{G}_{0}$ is orthogonal to $\mathbb{R}$. We denote the unit vector in $\mathbb{R}$ by $H_{0}$ and obtain a diffeomorphism $\phi: G_{0} \times \mathbb{R} \rightarrow G, \phi\left(g_{0}, t\right)=g_{0} \exp \left(t H_{0}\right)$.

Lemma 1. The left-invariant Haar measure $\mu$ on $G$ is given by

$$
d \mu\left(g_{0}, t\right)=\operatorname{det}\left(e^{\operatorname{ad}\left(-t H_{0}\right)}\right) d \nu\left(g_{0}\right) d t=e^{-t \operatorname{tr}\left(\operatorname{ad}\left(H_{0}\right)\right)} d \nu\left(g_{0}\right) d t,
$$

where $d \nu$ is the left-invariant Haar measure on $G_{0}=G_{0} \times\{0\} \subset G$.

Proof. Note that $d \nu d t$ is left $G_{0}$-invariant and right $\mathbb{R}$-invariant. Consequently, the left-invariant Haar measure on $G$ with respect to the above diffeomorphisms is given by $d \mu\left(g_{0}, t\right)=\delta(t) d \nu\left(g_{0}\right) d t$. We will calculate $\delta(t)$. Let $a=\exp \left(s H_{0}\right)$ and $f \in C_{0}^{\infty}(G)$. Then

$$
\begin{aligned}
\int_{G} f(a g) d \mu(g) & =\int_{\mathbb{R}} \int_{G_{0}} f\left(a g_{0} \exp \left(t H_{0}\right)\right) \delta(t) d \nu\left(g_{0}\right) d t \\
& =\int_{\mathbb{R}} \int_{G_{0}} f\left(\psi\left(g_{0}\right) a \exp \left(t H_{0}\right)\right) \delta(t) d \nu\left(g_{0}\right) d t
\end{aligned}
$$

where $\psi: G_{0} \rightarrow G_{0}, \psi\left(g_{0}\right)=a g_{0} a^{-1}$. From the transformation formula this becomes

$$
\int_{\mathbb{R}} \delta(t) \int_{G_{0}} f\left(g_{0} \exp \left((s+t) H_{0}\right)\right)\left|\operatorname{det} D \psi^{-1}\left(g_{0}\right)\right| d \nu\left(g_{0}\right) d t .
$$

Let $X_{1}, \cdots, X_{n}$ be an orthonormal basis of $\mathfrak{G}_{0}$ with respect to the left-invariant metric. Then the $Y_{j}=\left.\frac{d}{d t}\right|_{t=0} g_{0} \exp \left(t X_{j}\right), j=1, \ldots, n$, form an orthonomal basis of $T_{g_{0}} G_{0}$, and

$$
\begin{aligned}
D \psi^{-1}\left(g_{0}\right)\left(Y_{j}\right) & =\left.\frac{d}{d t}\right|_{t=0} \psi^{-1}\left(g_{0} \exp \left(t X_{j}\right)\right)=\left.\frac{d}{d t}\right|_{t=0} a^{-1} g_{0} \exp \left(t X_{j}\right) a \\
& =d L_{a^{-1} g_{0} a}\left(e^{\operatorname{ad}\left(-s H_{0}\right)} X_{j}\right) .
\end{aligned}
$$

So we conclude that $\left|\operatorname{det}\left(D \psi^{-1}\left(g_{0}\right)\right)\right|=\operatorname{det}\left(e^{\text {ad }\left(-s H_{0}\right)}\right)$. By left invariance of $\mu$ we get

$$
\begin{aligned}
\int_{G} f(a g) d \mu(g) & =\int_{\mathbb{R}} \delta(t) \operatorname{det}\left(e^{\operatorname{ad}\left(-s H_{0}\right)}\right) \int_{G_{0}} f\left(g_{0} \exp \left((s+t) H_{0}\right)\right) d \nu\left(g_{0}\right) d t \\
=\int_{G} f(g) d \mu(g) & =\int_{\mathbb{R}} \delta(s+t) \int_{G_{0}} f\left(g_{0} \exp \left((s+t) H_{0}\right)\right) d \nu\left(g_{0}\right) d t ;
\end{aligned}
$$

hence $\delta(s)=\operatorname{det}\left(e^{\operatorname{ad}\left(-s H_{0}\right)}\right)$.

The following theorem contains the main result of this section.

Theorem 3. For the Cheeger constant of a Lie group $G$ as above we have

$$
h(G) \geq\left|\operatorname{tr}\left(\operatorname{ad}\left(H_{0}\right)\right)\right| .
$$

Moreover, if $h\left(G_{0}\right)=0$, then formula (4) holds with equality. 
Proof. We identify $G$ with $G_{0} \times \mathbb{R}$ via the diffeomorphism $\phi$ and denote by $\pi: G \rightarrow$ $G_{0}$ the projection. Without loss of generality, we may assume that $\operatorname{tr}\left(\operatorname{ad}\left(H_{0}\right)\right) \geq 0$. For a connected, compact subset $K \subset G$ with smooth boundary $\partial K$ and nonempty interior, let $U=\pi(K) \backslash\left\{\right.$ critical values of $\left.\left.\pi\right|_{\partial K}\right\}$.

On $U$ we define functions $\delta^{ \pm}$by $\delta^{+}(u)=\max \{t \mid(u, t) \in K\}$ and $\delta^{-}(u)=$ $\min \{t \mid(u, t) \in K\}, u \in U$. The functions $\delta^{ \pm}$are smooth, and by Sard's theorem the set $U$ has full measure in $\pi(K)$. We denote by $\partial K^{ \pm}=\left\{\left(u, \delta^{ \pm}(u)\right) \mid u \in U\right\}$ the graphs of $\delta^{ \pm}$. We estimate the volumes of $K$ and $\partial K$. From (2) we get

$$
\begin{aligned}
\operatorname{vol}(K) & \leq \int_{U} \int_{\delta^{-}(u)}^{\delta^{+}(u)} e^{-t \operatorname{tr}\left(\operatorname{ad}\left(H_{0}\right)\right)} d t d \nu(u) \\
& \leq \frac{1}{\operatorname{tr}\left(\operatorname{ad}\left(H_{0}\right)\right)} \int_{U}\left(e^{-\delta^{+}(u) \operatorname{tr}\left(\operatorname{ad}\left(H_{0}\right)\right)}+e^{-\delta^{-}(u) \operatorname{tr}\left(\operatorname{ad}\left(H_{0}\right)\right)}\right) d \nu(u) .
\end{aligned}
$$

Clearly area $(\partial K) \geq \operatorname{area}\left(\partial K^{+}\right)+\operatorname{area}\left(\partial K^{-}\right)$. We have

$$
\operatorname{area}\left(\partial K^{+}\right)=\int_{U} \sqrt{\operatorname{det}\left(\left\langle D \varphi(u) e_{i}, D \varphi(u) e_{j}\right\rangle_{\left(u, \delta^{+}(u)\right)}\right)_{i, j=1 \ldots n-1}} d \nu(u),
$$

where $\varphi(u)=\left(u, \delta^{+}(u)\right)$ and $e_{1}, \ldots, e_{n-1}$ is an orthonormal basis of $T_{u} G_{0}$. We estimate the integrand

$$
\begin{aligned}
\sqrt{\operatorname{det}\left(\left\langle D \varphi(u) e_{i}, D \varphi(u) e_{j}\right\rangle_{\left(u, \delta^{+}(u)\right)}\right)} & =\sqrt{\operatorname{det}\left(\left\langle e_{i}, e_{j}\right\rangle_{\left(u, \delta^{+}(u)\right)}+v v^{\top}\right)} \\
& \geq \sqrt{\operatorname{det}\left(\left\langle e_{i}, e_{j}\right\rangle_{\left(u, \delta^{+}(u)\right)}\right)} \\
& =e^{-\delta^{+}(u) \operatorname{tr}\left(\operatorname{ad}\left(H_{0}\right)\right)}
\end{aligned}
$$

where $v^{\top}=\left(e_{1}\left(\delta^{+}\right), \ldots, e_{n-1}\left(\delta^{+}\right)\right)$and the $e_{i}$ 's on the right-hand side are considered as elements in $T_{\left(u, \delta^{+}(u)\right)} G$. The equality (5) follows from (2). This together with the analogous estimate for $\partial K^{-}$yields

$$
\operatorname{area}(\partial K) \geq \operatorname{tr}\left(\operatorname{ad}\left(H_{0}\right)\right) \operatorname{vol}(K) .
$$

This proves inequality (4).

Finally, we prove equality in (4) in the case $h\left(G_{0}\right)=0$. As before, we use the diffeomorphism $G \cong G_{0} \times \mathbb{R}$. Let $K_{0} \subset G_{0}$ be arbitrary and consider the set $K=K_{0} \times[a, b] \subset G$ with boundary $\partial K=\left(K_{0} \times\{a, b\}\right) \cup\left(\partial K_{0} \times[a, b]\right)$. Direct calculations yield

$$
\operatorname{vol}(K)=\operatorname{vol}\left(K_{0}\right) \frac{e^{-a \operatorname{trad} H_{0}}-e^{-b \operatorname{trad} H_{0}}}{\operatorname{trad} H_{0}},
$$

as well as

$$
\operatorname{area}\left(K_{0} \times\{a, b\}\right)=\operatorname{vol}\left(K_{0}\right)\left(e^{-a \operatorname{trad} H_{0}}+e^{-b \operatorname{trad} H_{0}}\right) .
$$

Given $\epsilon>0$, we can arrange for

$$
\frac{\operatorname{area}\left(K_{0} \times\{a, b\}\right)}{\operatorname{vol}(K)} \leq \operatorname{tr} \operatorname{ad} H_{0}+\epsilon
$$

by choosing $b$ sufficiently large. Note that (7) and the estimate (8) also make sense if $\operatorname{tr} \operatorname{ad} H_{0}=0$. 
Let $n=\operatorname{dim} \mathfrak{G}_{0}$. Choosing an orthonormal basis $e_{1}, \ldots, e_{n-1}$ of $T_{u} \partial K_{0} \subset \mathfrak{G}_{0}$ and abbreviating $e^{-t\left(\text { ad } H_{0}\right)}=A_{t}$, we finally compute

$$
\operatorname{area}\left(\partial K_{0} \times[a, b]\right)=\int_{a}^{b} \operatorname{vol}_{n-1}\left(\partial K_{0} \times\{t\}\right) d t,
$$

where the $(n-1)$-dimensional volume is given by

$$
\operatorname{vol}_{n-1}\left(\partial K_{0} \times t\right)=\int_{\partial K_{0}} \sqrt{\operatorname{det}\left(\left\langle A_{t} e_{p}, A_{t} e_{q}\right\rangle_{\mathfrak{G}_{0}}\right)_{p, q=1 \ldots n-1}} d \operatorname{vol}_{\partial K_{0}}(u) .
$$

The integrand can be estimated from above by

$$
M:=\max \left\{\sqrt{\operatorname{det}\left(\left.P_{U} A_{t}{ }^{*} A_{t}\right|_{U}\right)} \mid a \leq t \leq b, U \subset \mathfrak{G}_{0}, \operatorname{dim} U=n-1\right\},
$$

where $P_{U}: \mathfrak{G}_{0} \rightarrow U$ denotes orthogonal projection. Note that $M$ is independent of the specific choice of $K_{0}$. Therefore, choosing $K_{0}$ such that $\operatorname{vol}_{n-1} \partial\left(K_{0}\right) / \operatorname{vol}\left(K_{0}\right)$ is sufficiently small, we may assume that

$$
\frac{\operatorname{area}\left(\partial K_{0} \times[a, b]\right)}{\operatorname{vol}(K)} \leq \frac{M\left(\operatorname{trad} H_{0}\right)(b-a)}{e^{-a \operatorname{trad} H_{0}}-e^{-b \operatorname{trad} H_{0}}} \frac{\operatorname{vol}_{n-1}\left(\partial K_{0}\right)}{\operatorname{vol}\left(K_{0}\right)} \leq \epsilon .
$$

Putting (8) and (91) together yields $h(G)=\operatorname{tr}$ ad $H_{0}$, since $\epsilon>0$ was chosen arbitrarily small.

Now we consider the particular case of a simply connected solvable Lie group $G$ with Lie algebra $\mathfrak{G}$ and $\mathfrak{N}=[\mathfrak{G}, \mathfrak{G}]$. In this case we obtain

Corollary 10. The Cheeger constant of a simply connected solvable Lie group $G$ with Lie algebra $\mathfrak{G}$ is

$$
h(G)=\max _{H \in \mathfrak{G},\|H\|=1} \operatorname{tr}(\operatorname{ad}(H)) .
$$

Proof. Let $\mathfrak{G}_{0}$ denote the kernel of the 1-form $\alpha: \mathfrak{G} \rightarrow \mathbb{R}, X \mapsto \operatorname{tr}$ ad $X$. Clearly $\mathfrak{G}_{0}$ contains $\mathfrak{N}=[\mathfrak{G}, \mathfrak{G}]$, since $\mathfrak{N}$ is nilpotent. Hence $\mathfrak{G}_{0}$ is an ideal, and the corresponding unimodular Lie group satisfies $h\left(G_{0}\right)=0$ by Theorem 3, which proves the corollary in the case $\mathfrak{G}_{0}=\mathfrak{G}$. Otherwise $\mathfrak{G}_{0}$ has codimension 1 . Let $H_{0}$ be the maximum of $\alpha$ on the unit sphere of $\mathfrak{G}$, i.e.,

$$
\max _{H \in \mathfrak{G},\|H\|=1} \operatorname{tr}(\operatorname{ad}(H))=\operatorname{tr}\left(\operatorname{ad}\left(H_{0}\right)\right) .
$$

Then $H_{0} \perp \mathfrak{G}_{0}$ and, again, Theorem 3 implies the statement of the corollary.

Remarks. Note that simply connected, solvable Lie groups with left-invariant metric may have curvature of both signs (e.g., horospheres in symmetric spaces). Corollary 10]in the particular case of simply connected strictly negatively curved homogeneous spaces (NCHS) was proved by Connell $\mathrm{Co}$. He also showed for NCHS that the Cheeger constant coincides with the exponential volume growth rate.

Formula (11) also gives a lower bound for the topological entropy of compact Riemannian manifolds whose universal covering is a solvable Lie group with leftinvariant metric. This follows from the lower estimate of the topological entropy by the exponential volume growth rate proved in $\mathrm{Ma}$. In the case of a locally symmetric space $M$ the topological entropy was calculated in $[\mathrm{Sp}$ and agrees with both the exponential volume growth rate and the Cheeger constant of the universal covering $\tilde{M}$. It is given by $\|\rho\|$, where $\rho$ is the sum of the positive roots with 
multiplicities. More refined volume growth calculations in symmetric spaces were carried out in $\mathrm{Kn}$.

\section{ACKNOWLEDGMENTS}

We are grateful to P. Pansu for bringing to our attention the paper of H. Hoke. We thank the University of Cyprus for financial support.

\section{REFERENCES}

[Br] R. Brooks, The spectral geometry of a tower of coverings, J. Differential Geom. 23, 97-107, 1986. MR 87j:58095

[BPP] R. Brooks, P. Perry, and P. Petersen, On Cheeger's inequality, Comment. Math. Helvetici 68, 599-621, 1993. MR 94k:58150

[Bu] P. Buser, A note on the isoperimetric constant, Ann. Sci. École Norm. Sup. 15, 213-230, 1982. MR 84e:58076

[Cha] I. Chavel, Eigenvalues in Riemannian geometry, Academic Press, Orlando, FL, 1984. MR 86g:58140

[Che] J. Cheeger, A lower bound for the smallest eigenvalue of the Laplacian, "Problems in Analysis", pp. 195-199, Princeton Univ. Press, Princeton, NJ, 1970. MR 53:6645

[Co] Ch. Connell, Asymptotic harmonicity of negatively curved homogeneous spaces and their measures at infinity, Comm. Anal. Geom., vol. 8, no. 3, 575-633, 2000. MR 2001j:53061

[Gr] A. Grigor'yan, Estimates of heat kernels on Riemannian manifolds, "Spectral theory and geometry", pp. 140-225, Cambridge Univ. Press, Cambridge, 1999. MR 2001b:58040

[Ho] H. F. Hoke III, Lie groups that are closed at infinity, Trans. Amer. Math. Soc. 313, Number 2, 721-735, 1989. MR 89j:58108

[Kn] G. Knieper, On the asymptotic geometry of nonpositively curved manifolds, Geom. Funct. Anal. 7, 755-782, 1997. MR 98h:53055

[Leu] E. Leuzinger, Kazhdan's property $(T), L^{2}$-spectrum and isoperimetric inequalities for locally symmetric spaces, Comment. Math. Helv. 78, 116-133, 2003. MR 2004a:53047

[Ma] A. Manning, Topological entropy for geodesic flows, Ann. of Math. (2) 110, 567-573, 1979. MR 81e:58044

[Pa] A. Paterson, Amenability, Mathematical Surveys and Monographs, no. 29, Amer. Math. Soc., Providence, RI, 1988. MR 90e:43001

[Pe] N. Peyerimhoff, Isoperimetric and ergodic properties of horospheres in symmetric spaces, in A. Katok, R. de la Llave, Y. Pesin, H. Weiss, editors, Smooth ergodic theory and its applications, Proc. Sympos. Pure Math. 69, Amer. Math. Soc., Providence, RI, 2001, pp. 793-808. MR 2002i:37031

[Pi] Ch. Pittet, The isoperimetric profile of homogeneous Riemannian manifolds, J. Differential Geom. 54, 255-302, 2000. MR 2002g:53088

[Sp] R. J. Spatzier, Dynamical properties of algebraic systems, Dissertation, Warwick and Maryland, 1983.

Mathematische Fakultät, Ruhr-Universität Bochum, Universitätssstrasse 150, 44780 Bochum, Germany

E-mail address: peyerim@math.ruhr-uni-bochum.de

Department of Mathematics and Statistics, University of Cyprus, P.O. Box 20537, 1678 NiCOSIA, Cyprus

E-mail address: samiou@ucy.ac.cy 